PROCEEDINGS OF THE

AMERICAN MATHEMATICAL SOCIETY

Volume 131, Number 6, Pages 1881-1890

S 0002-9939(02)06742-4

Article electronically published on November 4, 2002

\title{
INVARIANT COMPLEMENTATION AND PROJECTIVITY IN THE FOURIER ALGEBRA
}

PETER J. WOOD

(Communicated by Andreas Seeger)

\begin{abstract}
AвSTRACT. In this paper, we study the ideals in the Fourier algebra of a locally compact group $G$ which are complemented by an invariant projection. In particular we show that when $G$ is discrete, every ideal which is complemented by a completely bounded projection must be invariantly complemented. Perhaps surprisingly, this result does not depend of the amenability of the group or the algebra, but instead relies on the operator biprojectivity of the Fourier algebra for a discrete group.
\end{abstract}

\section{INTRODUCTION}

The history of the problem of classifying the ideals in the Fourier algebra essentially goes back to D.J. Newman's result 9] in 1961 and more generally, Walter Rudin's paper [12] in 1962 where he classified the complemented ideals in the group algebra $\mathbf{L}^{1}(G)$, for compact abelian groups. Rudin used an averaging argument to show that an ideal $\mathcal{I}$ of $\mathbf{L}^{1}(G)$ is complemented if and only if there exists a projection $P: \mathbf{L}^{1}(G) \rightarrow \mathcal{I}$ which commutes with multiplication, i.e. $P(f * g)=f * P(g)$ for all $f, g \in \mathbf{L}^{1}(G)$. (We present a proof of this fact for compact non-abelian groups in Theorem 4.6.)

Using the Fourier transform, we can recognize Rudin's result in terms of the Fourier algebra $\mathbf{A}(G)$. Recall that the Fourier transform allows us to identify $\mathbf{L}^{1}(\hat{G})$ with $\mathbf{A}(G)$, where $\hat{G}$ is the dual group. Thus Rudin's result becomes: If $G$ is a discrete abelian group and $\mathcal{I}$ an ideal of $\mathbf{A}(G)$, then $\mathcal{I}$ is complemented if and only if there exists a projection $P: \mathbf{A}(G) \rightarrow \mathcal{I}$ such that $P(u v)=u P(v)$ for all $u, v \in \mathbf{A}(G)$. The main result of the current paper is to extend this result to the Fourier algebra of a non-abelian group.

It is easy to see from Rudin's construction that his result cannot be extended to non-compact groups. (See the discussion after Theorem 4.4.) However, H. Rosenthal 10 was able to address this problem for all amenable groups in the following way: If $\mathcal{I}$ is a complemented ideal of $\mathbf{L}^{1}(G)$, then there must exist a projection $Q: \mathbf{L}^{\infty}(G) \rightarrow \mathcal{I}^{\perp}$. Now since $G$ is amenable, we can use averaging arguments in $\mathbf{L}^{\infty}(G)$ to show that there must exist a projection $P: \mathbf{L}^{\infty}(G) \rightarrow \mathcal{I}^{\perp}$ such that $P(f * \phi)=f * P(\phi)$ for all $f \in \mathbf{L}^{1}(G)$ and $\phi \in \mathbf{L}^{\infty}(G)$ (i.e. $P$ commutes with the $\mathbf{L}^{1}(G)$ module action on $\left.\mathbf{L}^{\infty}(G)\right)$.

Received by the editors February 15, 2001 and, in revised form, February 8, 2002.

2000 Mathematics Subject Classification. Primary 43A30; Secondary 46L07.

Key words and phrases. Fourier algebra, operator space, projective, complemented ideals.

(C)2002 American Mathematical Society 
Let us rephrase these results in the following way: Consider the short exact sequences of $\mathbf{L}^{1}(G)$ modules

$$
(\Sigma): 0 \rightarrow \mathcal{I} \rightarrow \mathbf{L}^{1}(G) \rightarrow \mathbf{L}^{1}(G) / \mathcal{I} \rightarrow 0
$$

and its dual sequence

$$
\left(\Sigma^{*}\right): 0 \rightarrow \mathcal{I}^{\perp} \rightarrow \mathbf{L}^{\infty}(G) \rightarrow \mathcal{I}^{*} \rightarrow 0 .
$$

In the language of homological algebra (see $\$ 2$ for definitions), Rudin's result says that if $\mathcal{I}$ is complemented in $\mathbf{L}^{1}(G)$ for $G$ compact abelian, then $\Sigma$ splits. Rosenthal's result now reads: If $G$ is amenable and $\mathcal{I}$ is complemented in $\mathbf{L}^{1}(G)$, then $\Sigma^{*}$ splits. We now explore this question within this homological framework.

A Banach algebra $\mathcal{A}$ is said to be amenable if every bounded derivation into a dual $\mathcal{A}$ module is inner. In his important work [5], B. Johnson showed that $\mathbf{L}^{1}(G)$ is amenable if and only if $G$ is amenable. Using tools of homological algebra, Khelemskii was able to show in [7] that if the Banach algebra $\mathcal{A}$ is amenable, then the sequence of $\mathcal{A}$ modules

$$
(\Xi): 0 \rightarrow X^{*} \rightarrow Y \rightarrow Z \rightarrow 0
$$

splits, where $X^{*}$ is a dual $\mathcal{A}$ module. (See 2] for an alternate proof which avoids the homological algebra tools.)

Notice that the sequence $\Sigma^{*}$ is just a special case of a sequence given in $\Xi$. Assembling all this we realize that Rosenthal's results can now be obtained as follows: Let $G$ be amenable and $\mathcal{I}$ a complemented ideal in $\mathbf{L}^{1}(G)$. By Johnson's result $\mathbf{L}^{1}(G)$ is amenable, and by Khelemskii's work, the sequence $\Sigma^{*}$ must split. Thus there exists a projection $P: \mathbf{L}^{\infty}(G) \rightarrow \mathcal{I}^{\perp}$ which commutes with the $\mathbf{L}^{1}(G)$ module action. So it seems that it is the amenability of the algebra $\mathbf{L}^{1}(G)$ which is at the heart of the matter. In essence, it appears that the "averaging properties" are best captured at the algebra level.

It is the latter observation which drove the study of ideals in Fourier algebra. The initial hope was to proceed as follows: If $G$ is amenable, perhaps $\mathbf{A}(G)$ will be amenable. Thus whenever $\mathcal{I}$ is a complemented ideal in $\mathbf{A}(G)$, we can apply Khelemskii's results to construct a projection $P: \mathbf{A}(G)^{*} \rightarrow \mathcal{I}^{\perp}$ which commutes with the $\mathbf{A}(G)$ module action. Unfortunately this approach has failed. Johnson provided an example of a compact group $G$ for which the Fourier algebra $\mathbf{A}(G)$ is not amenable.

Fortunately, a new path was opened for us when Z-J Ruan introduced the notion of operator amenability (see $\S 2$ for definitions). In [11, Ruan showed that $\mathbf{A}(G)$ was operator amenable exactly when $G$ is amenable, thus establishing the natural analogue of Johnson's theorem for the group algebra. Exploiting this fact, the current author in 13. established analogous results to Khelemskii's to conclude: If $G$ is amenable and $\mathcal{I}$ is an ideal which is complemented by a completely bounded projection, then there exists a completely bounded projection $P: \mathbf{A}(G)^{*} \rightarrow \mathcal{I}^{\perp}$ which commutes with the $\mathbf{A}(G)$ module action. (See [14] for a more "homological" proof of this fact.) Thus we have established the analogue of Rosenthal's result for $\mathbf{A}(G)$ in the category of operator spaces.

Reconsidering Rudin's original result for compact abelian $G$, he showed that there is a projection $P: \mathbf{L}^{1}(G) \rightarrow \mathcal{I}$ which commutes with the module action. This appears to be a stronger result than Rosenthal's where the invariant projection acts on the dual space. This leads us to the analogous question in $\mathbf{A}(G)$ : If $G$ is 
discrete and $\mathcal{I}$ is complemented, does there exist a projection $P: \mathbf{A}(G) \rightarrow \mathcal{I}$ which commutes with the $\mathbf{A}(G)$ module action? In this paper, we answer this question in the affirmative in the category of operator spaces. Perhaps most surprisingly, this fact is unrelated to the amenability of the group $G$ or the algebra $\mathbf{A}(G)$. Instead, the result depends on the projectivity of the Fourier algebra for discrete groups.

\section{Definition And notation}

Let $G$ be a locally compact group, and let $\mathbf{L}^{1}(G)$ denote the group algebra. We let $\mathbf{A}(G)$ denote the Fourier algebra of $G$. If $G$ is abelian, then $\mathbf{A}(G)$ is simply the Fourier transform of the group algebra of $\hat{G}$. A $(G)$ was introduced for non-commutative groups by Eymard in [4]. In any case, $\mathbf{A}(G)$ is a subspace of $C_{0}(G)$, the space of continuous functions vanishing at infinity. We highlight the very important fact that if $G$ is not abelian, then $\hat{G}$ is not a group and thus there is no way for us to have an isomorphism between $\mathbf{A}(G)$ and the group algebra of $\hat{G}$. For each closed set $E \subset G$ we define the subspace $\mathcal{I}(E)$ as follows:

$$
\mathcal{I}(E)=\{u \in \mathbf{A}(G): u(x)=0 \text { for all } x \in E\} .
$$

It is easy to see that $\mathcal{I}(E)$ is a closed ideal in $\mathbf{A}(G)$. In addition, given any ideal $\mathcal{I} \subset \mathbf{A}(G)$, we define the $\boldsymbol{h u l l}$ of $\mathcal{I}$, denoted by $h(\mathcal{I})$, as follows:

$$
h(\mathcal{I})=\{x \in G: u(x)=0 \text { for all } u \in \mathcal{I}\} .
$$

It is easy to see that $h(\mathcal{I}(E))=E$. If $\mathcal{I}(E)$ is the only ideal whose hull is $E$, we call $E$ an S-set. It is well known that if $G$ is discrete, then every subset is an S-set. Let $\mathbf{V N}(G)$ denote the closure of $\mathbf{L}^{1}(G)$, considered as an algebra of convolution operators on $\mathbf{L}^{2}(G)$, with respect to the weak operator topology on $B\left(\mathbf{L}^{2}(G)\right)$. The von Neumann algebra $\mathbf{V N}(G)$ can be identified with the Banach space dual of $\mathbf{A}(G)$ 4. An operator space is a vector space $V$ together with a family \|\|$_{n}$ of Banach space norms (called operator space norms) on $\mathbb{M}_{n}(V)$, the space of $n \times n$ matrices with entries in $V$ such that

$$
\text { (i) }\left\|\left[\begin{array}{cc}
A & 0 \\
0 & B
\end{array}\right]\right\| \|_{n+m}=\max \left\{\|A\|_{n},\|B\|_{m}\right\}
$$

for each $A \in \mathbb{M}_{n}(V), B \in \mathbb{M}_{m}(V)$, and

$$
\text { (ii) }\left\|\left(\left[a_{i j}\right]\right) A\left(\left[b_{i j}\right]\right)\right\|_{n} \leq\left\|\left[a_{i j}\right]\right\|\|A\|_{n}\left\|\left[b_{i j}\right]\right\|
$$

for each $\left[a_{i j}\right],\left[b_{i j}\right] \in \mathbb{M}_{n}(\mathbb{C})$ and $A \in \mathbb{M}_{n}(V)$. Let $X$ and $Y$ be operator spaces and let $T: X \mapsto Y$. For each $n \in \mathbb{N}$ define

$$
T^{(n)}: \mathbb{M}_{n}(X) \mapsto \mathbb{M}_{n}(Y)
$$

by

$$
T^{(n)}\left[x_{i j}\right]=\left[T x_{i j}\right]
$$

The map $T$ is said to be completely bounded (or simply c.b. for short) if $\sup \left\{\left\|T^{(n)}\right\|\right\}<\infty$. We say that $T$ is a complete isometry if each $T^{(n)}$ is an isometry and that $T$ is a complete contraction if each $T^{(n)}$ is a contraction. We say that two operator spaces $X$ and $Y$ are $\boldsymbol{c . b}$. isomorphic if there exists a c.b. map $T: X \mapsto Y$ such that $T^{-1}$ is also completely bounded. Furthermore we shall 
say that $X$ and $Y$ are $\boldsymbol{c . b}$. isometrically isomorphic if the map $T$ can be chosen to be a complete isometry. For the Hilbert space $H$, we let

$$
H^{(n)}=\underbrace{H \oplus \cdots \oplus H}_{n} .
$$

Since there is a canonical identification between $\mathbb{M}_{n}(B(H))$ and $B\left(H^{(n)}\right)$, it is easy to show that $B(H)$ (and hence any closed subspace) is an operator space. A fundamental result in the theory is that every operator space is completely isometrically isomorphic to a norm closed subspace $S$ of $B(H)$, the algebra of bounded operators on the Hilbert space $H$, where the operator space structure on $S$ is the structure inherited from $B(H)$ ([3]). If $X$ is an operator space, then we can recognize $X^{*}$ as an operator space called the standard dual of $X$ (see [1]). For operator spaces $X, Y$ and $Z$, we call a bilinear map $T: X \times Y \mapsto Z$ jointly completely bounded, if for $\left[x_{i j}\right] \in \mathbb{M}_{n}(X)$ and $\left[y_{k l}\right] \in \mathbb{M}_{m}(Y)$ we have that

$$
\|T\|_{j c b}=\sup \left\{\left\|\left[T\left(x_{i j}, y_{k l}\right)\right]\right\|_{m n}:\left\|\left[x_{i j}\right]\right\|_{n} \leq 1,\left\|\left[y_{k l}\right]\right\|_{m} \leq 1\right\}
$$

is finite. There is an operator space analogue of the projective tensor product which we denote $X \hat{\otimes} Y$ such that

$$
J C B(X, Y ; Z)=C B(X \hat{\otimes} Y, Z) .
$$

That is to say, each jointly completely bounded map extends to a unique map on this operator space projective tensor product.

An associative algebra $\mathcal{A}$ which is also an operator space and is such that the multiplication

$$
m: \mathcal{A} \hat{\otimes} \mathcal{A} \mapsto \mathcal{A}
$$

is completely contractive is called a completely contractive Banach algebra. The Fourier algebra can be given a natural operator structure by virtue of it being the predual of a von Neumann algebra. In this case, this operator space structure results in a completely contractive Banach algebra (see [1] and [1]). A left Banach $\mathcal{A}$-module is a left $\mathcal{A}$-module $X$ that is itself a Banach space and for which

$$
\|a x\|_{X} \leq\|a\|_{\mathcal{A}}\|x\|_{X}
$$

for each $a \in \mathcal{A}$ and each $x \in X$. A right and two-sided Banach $\mathcal{A}$ module is defined analogously. We call a two-sided module a bimodule. If $X$ is a left Banach $\mathcal{A}$-module, then $X^{*}$ becomes a right Banach $\mathcal{A}$-module with respect to the action

$$
(\phi a)(x)=\phi(a x) .
$$

We call $X^{*}$ a dual right Banach $\mathcal{A}$-module. Naturally we can define dual left and bimodules analogously. In the category of operator spaces there are two ways to define an operator module. In this paper we shall call an operator space $X$ which is a left Banach $\mathcal{A}$-module, a left operator $\mathcal{A}$-module (or simply left $\mathcal{A}$-module if no confusion arises) if the module map is completely contractive with respect to the operator projective tensor product, that is to say, the module map

$$
\pi_{X}: \mathcal{A} \hat{\otimes} X \mapsto X
$$

is completely contractive. Clearly we may define operator right and bimodules analogously. Furthermore if $X$ is an operator module, then $X^{*}$ becomes a dual operator module with the dual actions defined above. Suppose $X$ is a left operator 
$\mathcal{A}$-module and $Y$ an operator space. Then we may consider $X \hat{\otimes} Y$ as a left operator $\mathcal{A}$ module by

$$
a \cdot(x \otimes y)=a x \otimes y
$$

for $a \in \mathcal{A}, x \in X$ and $y \in Y$. It is clear that if $Y$ is a right operator module, then $X \hat{\otimes} Y$ becomes a right operator module in the analogous way.

A bounded (resp. c.b.) map $T: X \rightarrow Y$, where $X$ and $Y$ are left Banach (resp. operator) $\mathcal{A}$-modules is called a left module map if $T(a \cdot x)=a \cdot T(x)$ for all $a \in \mathcal{A}$ and $x \in X$. Right and bimodule maps are defined analogously.

The sequence of left Banach (resp. operator) modules

$$
(\Sigma): 0 \rightarrow X \stackrel{f}{\rightarrow} Y \stackrel{g}{\rightarrow} Z \rightarrow Z \rightarrow 0
$$

is called exact if $f$ is one-to-one, $g$ is onto and im $f=k e r g$ for bounded (resp. c.b.) module maps $f$ and $g$. The sequence is called admissible if there exist bounded (resp. c.b.) maps $F: Y \rightarrow X$ and $G: Z \rightarrow Y$ such that $F f=i d_{X}$ and $g G=i d_{Z}$. We call $F$ a left inverse for $f$ and $G$ a right inverse for $g$. We say the sequence $\Sigma$ splits if the maps $F$ and $G$ can be chosen to be module maps. We handle sequences of right and bimodules analogously. In general a bounded (resp. c.b.) module map $\phi: X \rightarrow Y$ between Banach (resp. operator) modules is called admissible if there exists a bounded (resp. c.b.) linear map $\theta: Y \rightarrow X$ such that $\phi \circ \theta=i d_{i m} \phi$. Note that $\theta$ need not be a module map.

Let $\mathcal{A}$ be a Banach (resp. completely contractive Banach) algebra, and $X$ an Banach (resp. operator) bimodule. A bounded (resp. c.b.) map $D: \mathcal{A} \rightarrow X$ is called a derivation if $D(a b)=a D(b)+D(a) b$ for all $a, b \in \mathcal{A}$. If $x \in X$, then the map $D_{x}: \mathcal{A} \rightarrow X$ defined by $D_{x}(a)=a x-x a$ is easily seen to be a derivation. Such derivations are called inner. We say $\mathcal{A}$ is amenable as a Banach algebra (resp. amenable as a completely contractive Banach algebra) if every bounded (resp. c.b.) derivation into a dual Banach (resp. operator) module is inner. Johnson's famous theorem asserts that the Banach algebra $\mathbf{L}^{1}(G)$ is amenable as a Banach algebra if and only if $G$ is an amenable group. Ruan's Theorem says that $\mathbf{A}(G)$ is amenable as a completely contractive Banach algebra if and only if $G$ is amenable.

\section{Projectivity and ideals}

The following definition is the operator space category analogue of the definition of projectivity. (See 8 for the Banach space category.) Unless otherwise noted, in this section $\mathcal{A}$ will denote a completely contractive Banach algebra, and $X, Y$ and $Z$ will denote operator $\mathcal{A}$-modules.

Definition 3.1. Let $X$ be a left operator $\mathcal{A}$ module. $X$ is called left projective if given any c.b. admissible surjection $\phi: Y \rightarrow Z$ and any c.b. module map $\theta: X \rightarrow$ $Z$, there exists a c.b. module map $\psi: X \rightarrow Y$ such that the following diagram commutes:

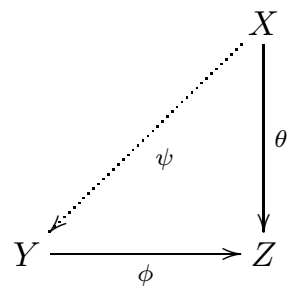


Clearly we can define right and biprojective in the analogous way. We say that the algebra $\mathcal{A}$ is operator biprojective if it is projective as an operator $\mathcal{A}$ bimodule.

The following is a standard property of projectivity which is easy to verify in the category of operator spaces.

Proposition 3.2. Let $Z$ be a projective $\mathcal{A}$-module and suppose

$$
\text { (П) }: 0 \rightarrow X \rightarrow Y \rightarrow Z \rightarrow 0
$$

is an admissible sequence of operator $\mathcal{A}$ modules. Then $\Pi$ splits.

Definition 3.3. Let $\mathcal{I}$ be a two-sided ideal in $\mathcal{A}$. We say $\mathcal{I}$ is complemented by $\boldsymbol{a}$ completely bounded projection (or simply complemented) if there exists a c.b. projection $P: \mathcal{A} \rightarrow \mathcal{I}$. Furthermore we shall say $\mathcal{I}$ is invariantly complemented if there exists a c.b. projection $P: \mathcal{A} \rightarrow \mathcal{I}$ such that $P(a b)=a P(b)=P(a) b$ for all $a, b \in \mathcal{A}$ (i.e. $P$ commutes with the module action).

We now have the following easy consequence of Proposition 3.2.

Theorem 3.4. Let $\mathcal{I}$ be a complemented two-sided ideal. Then $\mathcal{I}$ is invariantly complemented if $\mathcal{A} / \mathcal{I}$ is biprojective.

Proof. Consider the sequence

$$
(\Sigma): 0 \rightarrow \mathcal{I} \stackrel{i}{\rightarrow} \mathcal{A} \stackrel{q}{\rightarrow} \mathcal{A} / \mathcal{I} \rightarrow 0
$$

where $i$ is the inclusion map and $q$ is the canonical quotient map. Let $P: \mathcal{A} \rightarrow \mathcal{I}$ be a c.b. projection. So $P$ is a left inverse for $i$. First we show that $\Sigma$ is admissible. We define $Q: \mathcal{A} / \mathcal{I} \rightarrow \mathcal{A}$ by $Q(a+\mathcal{I})=a-P(a)$. Note that $Q$ is well defined since if $a+\mathcal{I}=b+\mathcal{I}$ we have $a-b \in \mathcal{I}$ so $Q(a-b+\mathcal{I})=a-b-P(a-b)=0$. Also note that $Q$ is completely bounded and is a right inverse for $q$. Thus $\Sigma$ is admissible. Hence by Proposition 3.2, $\Sigma$ splits. Thus there exists a c.b. module map $T: \mathcal{A} \rightarrow \mathcal{I}$ which is a left inverse for $i$. Hence $\mathcal{I}$ is invariantly complemented.

We now must determine under what conditions $\mathcal{A} / \mathcal{I}$ is biprojective. We begin with some definitions.

Let $\mathcal{A}_{+}$denote the algebra $\mathcal{A}$ with a unit attached. We denote this unit by $e$. $\mathcal{A}_{+}$can be given a natural operator space structure for which it is a completely contractive Banach algebra, and such that the natural inclusion $\mathcal{A} \rightarrow \mathcal{A}_{+}$is a complete isometry. (See 15].) Suppose we are given two operator $\mathcal{A}$-bimodules $X$ and $Y$. We define the tensor product $X \hat{\otimes}_{\mathcal{A}} Y$ as follows: Consider the operator subspace $N$ of $X \hat{\otimes} Y$ given by the closed linear span of elements of the form

$$
x a \otimes y-x \otimes a y .
$$

Now define $X \hat{\otimes}_{\mathcal{A}} Y$ by

$$
X \hat{\otimes}_{\mathcal{A}} Y=X \hat{\otimes} Y / N .
$$

We have the following facts which are generalizations of corresponding results in the category of Banach spaces. (See [15] for proofs.)

Proposition 3.5. Let $X$ be any operator $\mathcal{A}$ module, and let $P$ be a biprojective $\mathcal{A}$ bimodule. Then the modules $\mathcal{A}_{+} \hat{\otimes} X \hat{\otimes} \mathcal{A}_{+}$and $P \hat{\otimes}_{\mathcal{A}} X \hat{\otimes}_{\mathcal{A}} P$ are both biprojective. 
As an immediate consequence we have:

Theorem 3.6. Assume $\mathcal{A}$ is operator biprojective, and $\mathcal{I}$ is a complemented twosided ideal. If $\mathcal{A} \hat{\otimes}_{\mathcal{A}}(\mathcal{A} / \mathcal{I}) \hat{\otimes}_{\mathcal{A}} \mathcal{A}$ is c.b. isomorphic to $\mathcal{A} / \mathcal{I}$ as $\mathcal{A}$ bimodules, then $\mathcal{I}$ is invariantly complemented.

Proof. By Proposition 3.5, the module $\mathcal{A} \hat{\otimes}_{\mathcal{A}}(\mathcal{A} / \mathcal{I}) \hat{\otimes}_{\mathcal{A}} \mathcal{A}$ and hence $\mathcal{A} / \mathcal{I}$ is biprojective. Thus by Theorem $3.4, \mathcal{I}$ is invariantly complemented.

Now we shall investigate when the following holds: $\mathcal{A} \hat{\otimes}_{\mathcal{A}} \mathcal{A} / \mathcal{I} \hat{\otimes}_{\mathcal{A}} \mathcal{A}$ is c.b. isomorphic to $\mathcal{A} / \mathcal{I}$.

Definition 3.7. The algebra $\mathcal{A}$ will be called semi-neounital if the multiplication $\operatorname{map} M: \mathcal{A} \hat{\otimes} \mathcal{A} \rightarrow \mathcal{A}$ is onto.

Proposition 3.8. If $\mathcal{A}$ is semi-neounital, and $\mathcal{I}$ is any two sided ideal of $\mathcal{A}$, then there exists a c.b. isomorphism

$$
T: \mathcal{A} \hat{\otimes}_{\mathcal{A}}(\mathcal{A} / \mathcal{I}) \hat{\otimes}_{\mathcal{A}} \mathcal{A} \rightarrow \mathcal{A} / \mathcal{I}
$$

Proof. The proof of this fact is somewhat more complicated than it may appear at first glance. Consider the following diagram:

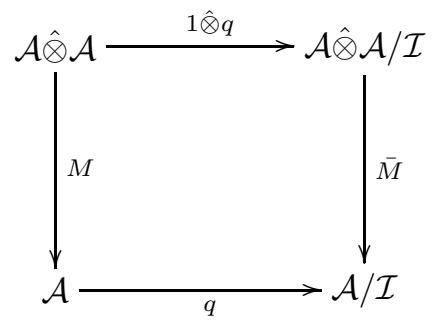

where $M$ is the usual multiplication map, $\bar{M}$ is the obvious module map, and $q$ the canonical quotient map. It is easy to see that the diagram commutes. Since $\mathcal{A}$ is semi-neounital, $M$ is onto. Since $q$ is onto, it follows that $\bar{M}$ must also be onto. Now consider the induced map $\tilde{M}: \mathcal{A} \hat{\otimes}_{\mathcal{A}} \mathcal{A} / \mathcal{I} \rightarrow \mathcal{A} / \mathcal{I}$. This map is clearly c.b. and onto as well. We must show that it is one-to-one and has a completely bounded inverse.

Let $M_{+}: \mathcal{A}_{+} \hat{\otimes}_{\mathcal{A}} \mathcal{A} \rightarrow \mathcal{A}$ be the multiplication map. It is easy to show that $\mathcal{A}_{+} \hat{\otimes}_{\mathcal{A}} \mathcal{A} / \mathcal{I}$ is completely isometrically isomorphic to $\mathcal{A} / \mathcal{I}$ via $M_{+}$, and that the inclusion map $\mathcal{A} \hat{\otimes}_{\mathcal{A}} \mathcal{A} / \mathcal{I} \rightarrow \mathcal{A}_{+} \hat{\otimes}_{\mathcal{A}} \mathcal{A} / \mathcal{I}$ is an isometry. (See [15.) Hence it follows that $\tilde{M}$ is one-to-one. Since the map $M_{+}$is a c.b. isomorphism and $\tilde{M}=\left.M_{+}\right|_{\mathcal{A} \hat{\otimes}_{\mathcal{A}} \mathcal{A} / \mathcal{I}}$ is bijective, it follows that $\tilde{M}$ is also a c.b. isomorphism. Also note that it is easy to see that $\tilde{M}$ is a left module map. Hence we conclude that $\mathcal{A} \hat{\otimes}_{\mathcal{A}} \mathcal{A} / \mathcal{I}$ and $\mathcal{A} / \mathcal{I}$ are c.b. isomorphic as left $\mathcal{A}$-modules.

Repeating these arguments, it follows that $\mathcal{A} / \mathcal{I}_{\hat{\otimes}} \mathcal{A} \mathcal{A}$ and $\mathcal{A} / \mathcal{I}$ are c.b. isomorphic via the "right" map $\tilde{M}_{R}$. Combining these results, we see that the map $\tilde{M}_{R}{ }^{\circ}\left(\tilde{M} \hat{\otimes}_{\mathcal{A}} 1\right)$ is a bimodule isomorphism of $\mathcal{A} \hat{\otimes}_{\mathcal{A}}(\mathcal{A} / \mathcal{I}) \hat{\otimes}_{\mathcal{A}} \mathcal{A}$ onto $\mathcal{A} / \mathcal{I}$.

The following technical lemma is a direct generalization of the analogous result in the Banach space category, so we omit the proof. (See [8, Proposition IV.5.4].)

Lemma 3.9. If $\mathcal{A}$ is biprojective, then $\mathcal{A}^{2}$ is dense in $\mathcal{A}$. 
Lemma 3.9 can be strengthened to the following:

Theorem 3.10. If $\mathcal{A}$ is biprojective, then $\mathcal{A}$ is semi-neounital.

Proof. Consider the following diagram:

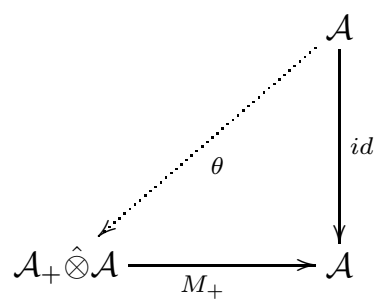

where $M_{+}$us the multiplication map and $i d$ the identity map. Note that the map $a \rightarrow e \otimes a$ is a completely bounded right inverse for $M_{+}$. (See [14.) Thus $M_{+}$is admissible.

Since $\mathcal{A}$ is biprojective we can find $\theta$ to make the diagram commute. We claim that $\operatorname{Im} \theta \subset \mathcal{A} \hat{\otimes} \mathcal{A}$. Since $\theta$ is a bimodule map, we have

$$
\theta(\mathcal{A} \cdot \mathcal{A}) \subset \mathcal{A} \cdot \theta(\mathcal{A}) \subset \mathcal{A} \cdot\left(\mathcal{A}_{+} \hat{\otimes} \mathcal{A}\right) \subset \mathcal{A} \hat{\otimes} \mathcal{A}
$$

Since $\overline{\mathcal{A}}^{2}=\mathcal{A}$ it follows by continuity that $\theta(\mathcal{A}) \subset \mathcal{A} \hat{\otimes} \mathcal{A}$. This generates the new commutative diagram:

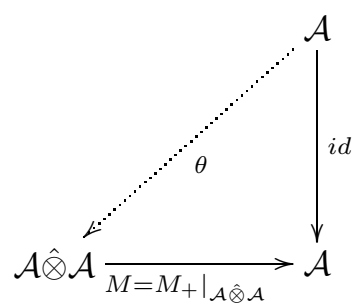

Since $i d$ is obviously onto, so is $M$. Hence $\mathcal{A}$ is semi-neounital.

Combining Proposition 3.8 and Theorem 3.10 with Theorem 3.6 we conclude:

Theorem 3.11. If $\mathcal{A}$ is biprojective, then every complemented two-sided ideal is invariantly complemented.

\section{Biprojectivity in Fourier and Group Algebras}

It is now a simple matter for us to apply the results of the last section to the Fourier algebra. We rely on the following results (see 15]):

Theorem 4.1. The Fourier algebra $\mathbf{A}(G)$ of a locally compact group $G$ is operator biprojective if and only if $G$ is discrete.

Combining Theorems 3.11 and 4.1 immediately gives the following:

Theorem 4.2. Let $G$ be a discrete group. An ideal $\mathcal{I}$ in $\mathbf{A}(G)$ is complemented if and only if it is invariantly complemented.

Definition 4.3. A function $f \in C(G)$ is called a multiplier of $\mathbf{A}(G)$ if $f \cdot u \in \mathbf{A}(G)$ for all $u \in \mathbf{A}(G)$. Furthermore, we call $f$ a completely bounded multiplier if the map $T_{f}: \mathbf{A}(G) \rightarrow \mathbf{A}(G)$ given by $T_{f}(u)=f \cdot u$ is completely bounded. We let

$$
\mathbf{A}(G)^{(c b)}=\left\{f \in C(G) \mid T_{f} \text { is completely bounded }\right\} .
$$


We now reach the following result:

Theorem 4.4. Let $G$ be discrete. Then the closed ideal $\mathcal{I}$ of $\mathbf{A}(G)$ is complemented by a completely bounded projection if and only if the function $1_{h(\mathcal{I})} \in \mathbf{A}(G)^{(c b)}$.

Proof. If $G$ is discrete, by Theorem 4.2 we have that $\mathcal{I}$ is invariantly complemented by a completely bounded projection, say $P: \mathbf{A}(G) \rightarrow \mathcal{I}$. Then we have $P(u v)=$ $u \cdot P(v)$. Hence there must exist a continuous function $f$ such that $P(u)=f \cdot u$. Since $P$ is a projection, $f$ is idempotent. Since $G$ is discrete, every subset of $G$ is an S-set, thus $\mathcal{I}=\mathcal{I}(h(\mathcal{I}))$. The fact that $f=1_{G \backslash h(\mathcal{I})}$ is now clear. Since $P$ is completely bounded, $f \in \mathbf{A}(G)^{(c b)}$.

The converse is obvious.

It not difficult to show that discreteness is essential in the above theorem. Suppose that every complemented ideal in $\mathbf{A}(G)$ is invariantly complemented. It is easy to see that the ideal $\mathcal{I}(\{e\})$ is a complemented ideal, hence is invariantly complemented (where $e$ denotes the identity element of the group $G$ ). Thus there exists a continuous function $f=1_{G \backslash\{e\}}$. Hence $G$ must be discrete.

Rudin's result for $L^{1}(G)$ dealt with ideals which were complemented by a bounded projection, not a completely bounded one. As a result, our Theorem 4.4 appears to be weaker than Rudin's theorem. However, as we shall see, this is in fact not the case.

Given any Banach space $X$, there exists a maximal operator space structure, denoted $M A X(X)$, such that if $Y$ is any operator space, and $T: X \rightarrow Y$, then $T: M A X(X) \rightarrow Y$ is automatically completely bounded. In particular, we get a functor $\mathcal{F}$ from Banach algebras to completely contractive Banach algebras. It is not hard to confirm that $X$ is biprojective as a Banach algebra if and only if $\mathcal{F}(X)$ is operator biprojective.

Now when $G$ is abelian, $\mathbf{A}(G)$ is the predual of a commutative operator algebra, and hence $\mathbf{A}(G)$ has the $M A X$ structure. Now since all bounded maps from $\mathbf{A}(G)$ to any operator space are automatically completely bounded, it follows that any ideal complemented by a bounded projection must be complemented by a completely bounded one. Thus we see that Theorem 4.4 is indeed a generalization of Rudin's result.

We wish to point out that our projectivity results can be applied to the group algebra $\mathbf{L}^{1}(G)$ as well. Note that for all groups $G, \mathbf{L}^{1}(G)$ is the predual of a commutative operator space, and hence it follows that the natural structure on $\mathbf{L}^{1}(G)$ is the MAX structure. Thus we can realize Khelemskii's theorem on the biprojectivity of $\mathbf{L}^{1}(G)$ as follows:

Theorem 4.5 ([8, Theorem IV.5.3]). $\mathbf{L}^{1}(G)$ is operator biprojective if and only if $G$ is compact.

The author suspects that the following result is well known (and indeed can be shown by more "classical" means); however he knows of no specific reference.

Theorem 4.6. Let $G$ be compact. The closed two-sided ideal $\mathcal{I}$ of $\mathbf{L}^{1}(G)$ is complemented if and only if there exists a central idempotent measure $\mu \in M(G)$ and a projection $P: \mathbf{L}^{1}(G) \rightarrow \mathcal{I}$ given by $P(f)=f * \mu$.

Proof. Since $G$ is compact, $\mathbf{L}^{1}(G)$ is operator biprojective. Thus $\mathcal{I}$ is invariantly complemented. Thus there exists $P: \mathbf{L}^{1}(G) \rightarrow \mathcal{I}$ where $P(f * g)=f * P(g)=$ 
$P(f) * g$ for all $f, g \in \mathbf{L}^{1}(G)$. Hence there exists a central measure $\mu$ such that $P(f)=f * \mu$. Since $P$ is a projection, $\mu$ is idempotent.

The converse is clear.

\section{REFERENCES}

[1] D.P Blecher, The standard dual of an operator space, Pacific J. Math. 153 (1992), 15-30. MR 93d:47083

[2] P.C. Curtis and R.J. Loy, Amenable Banach algebras, J. London Math. Soc. 40 (1989) 89-104. MR 90k:46114

[3] E.G. Effros and Z-J Ruan, On the abstract characterization of operator spaces, Proc. Amer. Math. Soc. 119 (1990), 579-584. MR 94g:46019

[4] P. Eymard, L'algebre de Fourier d'un groupe localemant compact, Bull. Soc. Math. France 92 (1964), 181-236. MR 37:4208

[5] B. Johnson, Cohomology in Banach Algebras, Mem. Amer. Math. Soc. 127 (1972). MR 51:11130

[6] , Nonamenability of the Fourier algebra for compact groups, J. London Math. Soc. 50 (1994), 361-374. MR 95i:43001

[7] A. Ya. Khelemskii, Flat Banach modules and amenable algebras, Trans. Moscow Math. Soc. (1984); Amer. Math. Soc. Translations (1985), 199-224. MR 86g:46108

[8] The Homology of Banach and Topological Algebras, Mathematics and its Applications (Soviet Series) 41, Kluwer Academic Publishers, 1986. MR 92d:46178

[9] D.J. Newman, The non-existence of projections from $L^{1}(\mathbb{T})$ to $H^{1}(\mathbb{T})$, Proc. Amer. Math. Soc. 12 (1961), 98-99. MR 22:11276

[10] H.P. Rosenthal, Projections onto translation invariant subspaces of $L^{p}(G)$, Mem. Amer. Math. Soc. 63 (1966), 84pp. MR 35:2080

[11] Z-J Ruan, The operator amenability of $A(G)$, Amer. J. Math. 117 (1995), 1449-1476. MR 96m:43001

[12] W. Rudin, Projections on invariant subspaces, Proc. Amer. Math. Soc. 13 (1962), 429-432. MR 25:1460

[13] P.J. Wood, Complemented ideals in the Fourier algebra of a locally compact group, Proc. Amer. Math. Soc. 128 No. 2 (2000), 445-451. MR 2000c: 43004

[14] - Homological Algebra in Operator Spaces with Applications to Harmonic Analysis, Ph.D. Thesis, University of Waterloo, 1999.

[15] _ The operator biprojectivity of the Fourier algebra, Can. J. Math (to appear).

Department of Pure Mathematics, University of Waterloo, Waterloo, Ontario, CANADA N2L 3G1

E-mail address: pwood@uwaterloo.ca 